ELECTRONIC RESEARCH ANNOUNCEMENTS OF THE AMERICAN MATHEMATICAL SOCIETY

Volume 12, Pages 1-12 (February 10, 2006)

S $1079-6762(06) 00154-5$

\title{
CLUSTER HOMOLOGY: AN OVERVIEW OF THE CONSTRUCTION AND RESULTS
}

\author{
OCTAV CORNEA AND FRANÇOIS LALONDE
}

(Communicated by Leonid Polterovich)

\begin{abstract}
We associate, to a Lagrangian submanifold $L$ of a symplectic manifold, a new homology, called the cluster homology of $L$, which is invariant up to ambient symplectic diffeomorphisms. We discuss various applications concerning analytical, topological, and dynamical properties of Lagrangian submanifolds. We also deduce a new universal Floer homology, defined without obstruction, for pairs of Lagrangian submanifolds.
\end{abstract}

\section{INTRODUCTION}

One of the main problems in symplectic topology is to manage efficiently bubbling for moduli spaces of pseudoholomorphic disks with boundary on a Lagrangian submanifold $L$ of a symplectic manifold $(M, \omega)$. One approach to this issue is provided by the work of Fukaya et al. [5]. In this announcement, we propose a solution to this problem based on different geometric and algebraic ideas 1

We assume here that all Lagrangian submanifolds are compact, connected, orientable, and relative spin (recall that a Lagrangian submanifold $L \subset(M, \omega)$ is relative spin if the second Stiefel-Whitney class of $L$ admits an extension to $H^{2}(M ; \mathbb{Z} / 2)$; a set of Lagrangian submanifolds is relative spin if their second Stiefel-Whitney classes admit a common extension to $H^{2}(M ; \mathbb{Z} / 2)$ ). In the notation $L$ for a Lagrangian submanifold we always implicitly include the choices of an orientation and of a relative spin structure (the same applies to a set of such submanifolds) as described in [5]. The ambient symplectic manifold $\left(M^{2 n}, \omega\right)$ is supposed to be compact or, if not, it should be geometrically bounded, so that no sequence of Riemann surfaces with boundary lying on a set of compact Lagrangian submanifolds $L_{1}, \ldots, L_{\ell} \subset M$ can escape to infinity. In fact, the Lagrangian submanifolds need not all be compact, as long as the above control on sequences of Riemann surfaces is ensured.

Received by the editors August 31, 2005 and, in revised form, October 10, 2005.

2000 Mathematics Subject Classification. Primary 53D12; Secondary 53D40.

Key words and phrases. Lagrangian submanifolds, bubbling.

The authors were supported in part by individual NSERC Discovery Grants and by team FQRNT grants.

${ }^{1}$ We have learned after the submission of this paper that the term "cluster" has been used in a number of other circumstances. To avoid any possible confusion we emphasize that the meaning we associate to this terminology is specific to the context of this paper.

(C)2006 American Mathematical Society Reverts to public domain 28 years from publication 
The key idea of our construction has its origin in the observation that the main difficulty in modelling algebraically the bubbling of disks is that this is a nonlocalized codimension-one phenomenon: not only does it produce boundary components but, in contrast with the usual breaking of Morse flow lines, the points where the bubbling appears are arbitrary. We tackle this problem by enlarging the moduli space of pseudoholomorphic disks by allowing configurations formed of disks connected by negative gradient flow lines of an a priori fixed Morse function on our Lagrangian submanifold. The resulting moduli spaces are called clustered moduli spaces. Their compactifications have boundaries consisting only of pairs of configurations joined at some critical point of the fixed Morse function. The combinatorics of these moduli spaces is then codified in a graded, commutative differential algebra over the rationals, which we call the cluster algebra and whose homology is called the cluster homology.

Once the cluster algebra is introduced, we define a universal Floer theory, the fine Floer homology, associated to the pair formed by two relative spin Lagrangian submanifolds $L, L^{\prime} \subset(M, \omega)$ in general position: it is the homology of a chain complex freely generated by (possibly, some of) the intersection points of $L$ and $L^{\prime}$ over a ring roughly of the form $\mathcal{C} \ell_{*}(L, f, J) \otimes \mathcal{C} \ell_{*}\left(L^{\prime}, f^{\prime}, J\right)$. The basic idea here is to use the cluster algebra as a rich coefficient ring over which Floer type constructions are possible despite the presence of bubbling.

In this paper we summarize these constructions and also mention a number of applications. The details of this work are contained in Cornea, Lalonde 4 except for the proof of the basic analytical structure of our moduli spaces, which is postponed to a later paper based on the Hofer-Wysocki-Zehnder's polyfold theory [9].

We thank Katrin Wehrheim for useful discussions.

\section{Definition And properties of the Cluster COMPleX}

The cluster complex is associated to a triple formed of (1) a Lagrangian embedding $L^{n} \hookrightarrow(M, \omega)$, equipped with a choice of an orientation and of a relative spin structure, (2) a generic almost complex structure $J$ on $M$ compatible with the symplectic form $\omega$, and (3) a pair $(f, g)$ with $f: L \rightarrow \mathbb{R}$ a Morse function and $g$ a Riemannian metric on $L$ so that $(f, g)$ is Morse-Smale. The two conditions implicit in the notation $L$ (i.e., the orientation and the relative spin structure) are needed to orient the clustered moduli spaces - to be roughly described below - in a coherent way.

This complex is denoted by $\mathcal{C} \ell_{*}(L ; J,(f, g))$ and, if $\operatorname{Crit}(f)$ is the set of critical points of $f$, we put

$$
\mathcal{C} \ell_{*}(L ; J,(f, g))=\left(S \mathbb{Q}\left\langle s^{-1} \operatorname{Crit}(f)\right\rangle \otimes \Lambda\right)_{*}^{\wedge},
$$

where $s^{-1} \operatorname{Crit}(f)$ indicates that the natural index grading of Crit $(f)$ is decreased by one unit, $S V$ is the free, graded commutative algebra over the graded vector space $V$ (as usual, the sign commutativity rule is $a b=(-1)^{|a||b|} b a$ for any two elements $a, b \in V)$, and $\Lambda$ is the rational group $\operatorname{ring}$ of the quotient $\pi_{2}(M, L) / \sim$, where the equivalence relation $\sim$ is given by $\lambda \sim \tau$ iff $\omega(\lambda)=\omega(\tau)$ and $\mu(\lambda)=\mu(\tau)$, for $\omega$ and $\mu$ the area and Maslov classes, respectively. We write the elements of $\Lambda$ in the form of finite sums $\sum_{i} c_{i} e^{\lambda_{i}}, c_{i} \in \mathbb{Q}$. The grading in $\Lambda$ is given by $\left|e^{\lambda}\right|=-\mu(\lambda)$ for $\lambda \in \pi_{2}(M, L) / \sim$. With this convention, the grading of the cluster complex is 
given by the usual tensor product formula. Thus for $x_{i} \in \operatorname{Crit}(f)$, we have

$$
\left|x_{i}\right|=\operatorname{ind}_{f}\left(x_{i}\right)-1,\left|x_{1} \cdots x_{k} e^{\lambda}\right|=\sum_{i=1}^{k}\left|x_{i}\right|-\mu(\lambda)
$$

Finally, we describe the completion ${ }^{\wedge}$. An element $m \in \mathcal{C} \ell(L ; J,(f, g))$ can be written as a possibly infinite sum

$$
m=m_{0}+m_{1} e^{\lambda_{1}}+\cdots+m_{i} e^{\lambda_{i}}+\cdots,
$$

where the $m_{i}$ are monomials in the elements of $\operatorname{Crit}(f)$ but, if this sum is infinite, then any infinite subsequence with $\omega\left(\lambda_{i}\right)$ bounded above must have its corresponding word length sequence converging to infinity (in the sense that any of its subsequences is unbounded) and additionally there is a Novikov type condition which states that any sum $\sum a_{\lambda} e^{\lambda}$ with $a_{\lambda}$ a formal series in the critical points of $f$ has the property that, for any $k \in \mathbb{R}$, there are only finitely many $a_{\lambda} \neq 0$ when $\omega(\lambda) \leq k$. Conversely, any formal sum satisfying these conditions belongs to the cluster complex.

We now give a rough idea of the construction of the cluster differential. Given the generic data $J,(f, g)$ on $L$, fix a linear order on the critical points of $f$. Choose any integer $k \geq 0$, any $x \in \operatorname{Crit}(f)$, any non-decreasing sequence of critical points $x_{1}, \ldots, x_{k}$, and $\lambda \in \pi_{2}(M, L) / \sim$, with the constraint that the zero class $\lambda=0$ is allowed only when $k$ equals 1 . The cluster differential

$$
d^{\nu}:\left(S \mathbb{Q}\left\langle s^{-1} \operatorname{Crit}(f)\right\rangle \otimes \Lambda\right)_{*}^{\wedge} \rightarrow\left(S \mathbb{Q}\left\langle s^{-1} \operatorname{Crit}(f)\right\rangle \otimes \Lambda\right)_{*-1}^{\wedge}
$$

is the unique commutative graded differential algebra extension of

$$
d^{\nu} x=\sum_{\lambda, k \geq 0, x_{1}, \ldots, x_{k}} a_{x_{1}, \ldots, x_{k}}^{x}(\lambda) x_{1} \cdots x_{k} e^{\lambda}
$$

where $x, x_{1}, \ldots, x_{k} \in \operatorname{Crit}(f)$ have the property that $\left(x_{1}, \ldots, x_{k}\right)$ respects the fixed order on $\operatorname{Crit}(f),|x|-\sum_{i}\left|x_{i}\right|+\mu(\lambda)=1$ and the coefficients $a_{x_{1}, \ldots, x_{k}}^{x}(\lambda)$ count with signs the number of elements in the clustered moduli spaces

$$
{ }^{\nu} \mathcal{M}_{x_{1}, \ldots, x_{k}}^{x}(\lambda)
$$

(due to the possible presence of multisections this number belongs in fact to $\mathbb{Q}$ ).

A rigorous description of the clustered moduli spaces appears in [4. The main idea in their definition is simple: consider a one-parameter family of $J$-disks of class $\lambda$ and assume that the bubbling off a $J$-disk of class $\lambda^{\prime}$ occurs in this family. The "bubbled" configuration formed by two touching $J$-disks of classes $\lambda^{\prime \prime}=\lambda-\lambda^{\prime}$ and $\lambda^{\prime}$ is then viewed as the limit of a one-parameter family of two $J$-disks joined by a negative gradient flow line of $f$ whose length tends to 0 and thus becomes an interior point of the larger clustered moduli space. This is represented schematically 
in the following picture:
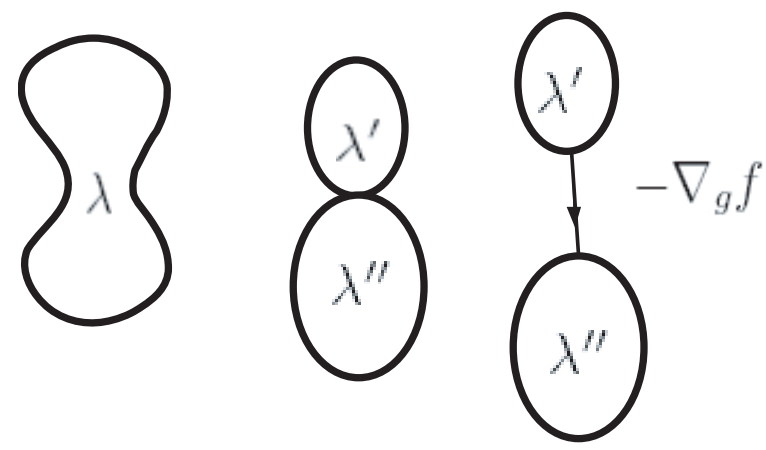

By pursuing systematically this idea together with the usual description of stable maps (as, for example, in McDuff-Salamon [10]) we obtain moduli spaces of configurations modelled on oriented trees with edges of non-negative length so that each vertex corresponds to a $J$-disk (or sphere), and each edge corresponds to a negative flow line of $f$ joining incidence points situated on the boundaries of the disks whose corresponding vertices are related by that edge. To insure a reasonable structure for these moduli spaces, we need to use a system $\nu$ of perturbations of the pseudoholomorphic equation. Moreover, to define ${ }^{\nu} \mathcal{M}_{x_{1}, \ldots, x_{k}}^{x}(\lambda)$, such a configuration is supposed to carry also $k+1$ additional marked points $z_{0}, \ldots, z_{k}$ so that $z_{0}$ belongs to the boundary of the root disk (which corresponds to the root vertex of the tree) and the $z_{i}$ belong to the boundaries of some of the disks involved so that $z_{0}$ is in the unstable manifold of the critical point $x$, and $z_{i}$ is in the stable manifold of $x_{i}$; the sum of the classes of the disks and spheres involved has to be $\lambda$. There are, of course, appropriate stability conditions. The role of ghost disks (for which the corresponding $J$-disk is constant) is particularly important as they allow to deal not only with the transversality issues due to multiple coverings but also with the crossing of some of the incidence or marked points.

The dimension of ${ }^{\nu} \mathcal{M}_{x_{1}, \ldots, x_{k}}^{x}(\lambda)$ equals

$$
|x|-\sum_{i=1}^{k}\left|x_{i}\right|+\mu(\lambda)-1
$$

These moduli spaces admit natural compactifications ${ }^{\nu} \overline{\mathcal{M}}_{x_{1}, \ldots, x_{k}}^{x}(\lambda)$. To describe their properties, we introduce the notation $S$ for a partially ordered set of critical points (where repetitions are allowed), which, of course, can be identified with a unique non-decreasing sequence of points. If $S^{\prime}, S^{\prime \prime}$ are two such ordered subsets, we will denote by $\left\langle S^{\prime} \cup S^{\prime \prime}\right\rangle$ the partially ordered subset made of the elements in $S^{\prime} \cup S^{\prime \prime}$. Letting $S$ be the ordered set $\left\{x_{1}, \ldots, x_{k}\right\}$, for those moduli spaces of dimension 1 (which are, in fact, branched 1-dimensional manifolds with rational weights) we have

$$
\partial\left({ }^{\nu} \overline{\mathcal{M}}_{S}^{x}(\lambda)\right)=\bigcup_{S=\left\langle S^{\prime} \cup S^{\prime \prime}\right\rangle, y, \lambda^{\prime}+\lambda^{\prime \prime}=\lambda}\left({ }^{\nu} \overline{\mathcal{M}}_{\left\langle S^{\prime}, y\right\rangle}^{x}\left(\lambda^{\prime}\right)\right) \times\left({ }^{\nu} \overline{\mathcal{M}}_{S^{\prime \prime}}^{y}\left(\lambda^{\prime \prime}\right)\right) .
$$

Here the summation is taken over all $y \in \operatorname{Crit}(f)$, all partitions of $S$ into two subsets $S^{\prime}, S^{\prime \prime}$, all splittings of $\lambda$ as a sum of two classes $\lambda^{\prime}, \lambda^{\prime \prime}$, and all the positions in which $y$ appears in $\left\langle S^{\prime}, y\right\rangle$ (this is relevant if $y$ is already present in $S^{\prime}$ because in this case this counts the number of possibilities of gluing at $y$ ). We also assume 
here that the set $S$ does not contain a repetition of a critical point $x$ of odd degree. This insures that ${ }^{\nu} \overline{\mathcal{M}}_{S}^{x}(\lambda)$ admits an orientation, and the equation is verified by taking into account orientations with certain signs before the terms on the righthand side, whereas the orientation on the left-hand side is clearly the one induced on the boundary.

Of course, by Gromov's compactification theorem, only a finite number of the moduli spaces appearing on the right-hand side of the last equation are non-empty.

This equation implies that the square of the cluster differential, as defined above, vanishes so that we deduce the first part of the result below.

Theorem 1.1. The map $d^{\nu}$ satisfies $\left(d^{\nu}\right)^{2}=0$. The resulting homology $\mathcal{C} \ell H_{*}(L)$ does not depend on the choices of $\nu,(f, g)$, and $J$ up to isomorphism.

Due to the commutative DGA setting, proving the invariance part of this statement is more delicate than just the usual Floer type construction for comparison morphisms (see again [4]). Note that this statement implies that $\mathcal{C} \ell H_{*}(L) \simeq$ $\mathcal{C} \ell H_{*}(\phi(L))$ for any symplectic diffeomorphism $\phi: M \rightarrow M$.

Remarks 1.2. a. A critical point $y$ of index 0 can never appear as an end in a 0-dimensional non-empty moduli space of type

$$
{ }^{\nu} \mathcal{M}_{\ldots, y, \ldots}^{x}(\lambda)
$$

(except for usual Morse flow lines). This happens because, if there were a negative gradient flow line from a non-constant loop in $L$ to a local minimum, there would be a one-parameter family of these lines (in our construction all $J$-spheres that might appear are attached to some non-trivial $J$-disk, which means that we may indeed assume the loop in question to be non-constant). Similarly, for such a moduli space $x$ cannot be a local maximum.

b. An element in the cluster complex, $\tau \in \mathcal{C} \ell(L ; J,(f, g))$, is written as a sum $\tau=\sum_{\lambda}(a(\lambda)+m(\lambda)) e^{\lambda}$, where $a(\lambda) \in \mathbb{Q}$ and $m(\lambda)$ is a sum of words (in the letters consisting of the critical points of $f$ ) of length at least one. We call each of the terms $a(\lambda) e^{\lambda}$ with $a(\lambda) \neq 0$ a free term of $\tau$. If there is a critical point $x$ of $f$ whose differential contains at least one free term, we say that the complex has free terms. Further, if the Morse index of $x$ above is larger than or equal to 1, we say that the cluster complex has high free terms. Note that, due to the fact that $\mu(\lambda)$ is even, if a critical point $x$ satisfies $d x=a_{0} e^{\lambda}+\cdots, a_{0} \neq 0$, then $\operatorname{ind}_{f}(x)$ is even. Moreover, in view of part a, $\operatorname{ind}_{f}(x) \neq \operatorname{dim}(L)$.

c. It is not difficult to verify that if the cluster complex is acyclic (that is, $\left.\mathcal{C} \ell H_{*}(L)=0\right)$, then the cluster has free terms. If the cluster complex $\mathcal{C} \ell(L, J, f)$ has high free terms, then, moreover, there are some $J$-disks of non-positive Maslov class (i.e., with Maslov index $\leq 0$ ).

Example 1.3. If $S^{1}$ is a circle in $\mathbb{C}$, we have

$$
\mathcal{C} \ell H_{*}\left(S^{1}\right)=0 \text {. }
$$

Indeed, take on $S^{1}$ the perfect Morse function with one minimum $m$ and one maximum $M$. There exists one pseudoholomorphic disk passing through $m$, of Maslov index 2, with a class in $\Lambda$ that we will denote by $\lambda_{0}$. For the maximum, we have $d M=0$. The differential of the minimum can be seen to be given by $d m=(1+M+s) e^{\lambda_{0}}$, where $s$ is a polynomial in $M$ without constant or linear terms. This implies the claim because in our ring we may then find a series $Q$ so that $Q(1+M+s)=1$, which means that $d\left(Q m e^{-\lambda_{0}}\right)=1$. 
Example 1.4. In the absence of bubbling (for example, if $\left.\omega\right|_{\pi_{2}(M, L)}=0$ ), we have

$$
\left.\mathcal{C} \ell H_{*}(L, J ;(f, g)) \simeq S\left(s^{-1} H_{*}(L ; \mathbb{Q})\right) \otimes \Lambda\right)^{\wedge} .
$$

This happens, of course, because in this case the only component of the cluster differential is provided by the usual Morse differential.

Remark 1.5. Spheres and disks with "spikes" have been used in various ways by other authors. Most notable is the announcement made by $\mathrm{Oh}$ in [11, which applies when the minimal Maslov number is larger than or equal to 2. Other instances are the work of Schwarz [15] as well as Piunikhin-Salamon-Schwarz [13] and more recently Bourgeois [3].

\section{Fine Floer homology}

The purpose of this section is to introduce a variant of Floer homology called fine Floer homology (denoted $\mathbb{F} H_{*}(-)$ ), which is associated to a pair of transversal orientable Lagrangian submanifolds $L_{0}, L_{1}$ of a symplectic manifold $(M, \omega)$, under the assumption that they be relative spin in $M$.

2.1. Coefficient ring and moduli spaces. To define the fine Floer complex

$$
\mathbb{F} C\left(L_{0}, L_{1}, \eta ; J,\left(f_{0}, g_{0}\right),\left(f_{1}, g_{1}\right)\right),
$$

we first recall that the choices of orientations and of a relative spin structure for $L_{0}, L_{1}$ have been made and are included in the notation $L_{0}, L_{1}$. Besides this, we need the following additional data. First, as before, we need an almost complex structure $J$, Morse-Smale pairs $\left(f_{i}, g_{i}\right)$ on $L_{i}$, and coherent choices of perturbations. We also assume that the $f_{i}$ are in general position with respect to the intersection points $L_{0} \cap L_{1}$ in the sense that these intersection points are included in the unstable manifolds of critical points of index 0 of $f_{i}$. We denote by $\Gamma=\{\alpha:[0,1] \rightarrow M$ : $\left.\alpha(i) \in L_{i}, i=0,1\right\}$ the space of continuous paths from $L_{0}$ to $L_{1}$. Here, $\eta$ is an element in $\Gamma$-its choice means that we fix a basepoint for this space. We denote by $\Gamma_{\eta}$ the connected component of $\Gamma$ which contains $\eta$. We denote by $I\left(L_{0}, L_{1}\right)$ the intersection points of $L_{0}$ and $L_{1}$ and we let $I_{\eta}$ be those intersection points which, viewed as constant paths, belong to $\Gamma_{\eta}$. The generators of the fine Floer complex will be precisely the elements of $I_{\eta}$. Up to a shift in degrees, the resulting fine Floer homology will only depend on $L_{0}, L_{1}$, the connected component of $\eta$, and the choice of orientations and relative spin structures of $L_{0}, L_{1}$.

To continue the construction, note that there are two group morphisms

$$
\omega: \pi_{1} \Gamma_{\eta} \rightarrow \mathbb{R}, \mu: \pi_{1} \Gamma_{\eta} \rightarrow \mathbb{Z}
$$

the first given by integration of $\omega$ and the second, a Maslov index type morphism, obtained in the usual way as in Robbin, Salamon [14.

We now define

$$
\mathcal{R}=\left(S \mathbb{Q}\left\langle s^{-1}\left(\operatorname{Crit}\left(f_{0}\right) \cup \operatorname{Crit}\left(f_{1}\right)\right)\right\rangle \otimes \bar{\Lambda}\right)^{\wedge},
$$

where $\bar{\Lambda}$ is the rational group ring of $\Pi=\operatorname{Im}(\omega \times \mu)$; the completion is as in the case of the cluster complex except that we take into consideration critical points of both $f_{0}$ and $f_{1}$.

Note that there are injective group morphisms $\phi_{i}: \pi_{2}\left(M, L_{i}\right) / \sim \rightarrow \Pi$, which are obtained by first assuming that $\eta$ joins the base points in $L_{0}$ and $L_{1}$ and then viewing a disk with boundary in, say, $L_{0}$ as a cylinder whose end on $L_{1}$ is constant. 
Therefore, if we denote by $\Lambda_{i}$ the group ring of $\pi_{2}\left(M, L_{i}\right) / \sim$, we have injective ring morphisms $\phi_{i}: \Lambda_{i} \rightarrow \bar{\Lambda}$. Thus, $\mathcal{R}$ is isomorphic to the obvious completion of

$$
\mathcal{C} \ell\left(L_{0}, f_{0}, J\right) \otimes \mathcal{C} \ell\left(L_{1}, f_{1}, J\right) \otimes_{\Lambda_{0} \otimes \Lambda_{1}} \bar{\Lambda} .
$$

2.2. The fine Floer complex. This is the free differential graded module over $\mathcal{R}$ given by

$$
\mathbb{F} C\left(L_{0}, L_{1}, \eta ; J,\left(f_{0}, g_{0}\right),\left(f_{1}, g_{1}\right)\right)=\left(\mathcal{R} \otimes \mathbb{Q}\left\langle I_{\eta}\right\rangle, d_{F}\right) .
$$

The grading of the elements in $I_{\eta}$ is obtained as follows: we consider lifts $\bar{a} \in \tilde{\Gamma}_{\eta}$ of the points $a \in I_{\eta} \subset \Gamma_{\eta}$, where $\tilde{\Gamma}_{\eta}$ is the regular covering of $\Gamma_{\eta}$ associated to $\Pi$ (here, as usual, the last inclusion means that we view intersection points as constant paths), and we define $|a|=\mu(\bar{a})$. This grading depends on the choice of the lifts, but different choices produce isomorphic complexes.

We now describe the differential $d_{F}$. We order the critical points in $\operatorname{Crit}\left(f_{i}\right)$. The differential $d_{F}$ satisfies the Leibniz formula and, for an element $a \in I_{\eta}$, it is of the form

$$
d_{F} a=\sum_{\lambda, b, k \geq 0, l \geq 0,\left(x_{1}, \ldots, x_{k}, y_{1} \cdots, y_{l}\right)} w_{x_{1}, \ldots, x_{k}, y_{1}, \ldots, y_{l} ; b}^{a}(\lambda) x_{1} \cdots x_{k} y_{1} \cdots y_{l} b e^{\lambda},
$$

where the $x_{i}$ belong to $\operatorname{Crit}\left(f_{0}\right)$, the $y_{j}$ to $\operatorname{Crit}\left(f_{1}\right)$, they respect the order, $\lambda \in \Pi$, and finally $b \in I_{\eta}$.

The coefficients $w_{x_{1}, \ldots, x_{k}, y_{1}, \ldots, y_{l} ; b}^{a}(\lambda) \in \mathbb{Q}$ count the number of elements in certain 0 -dimensional moduli spaces $\mathcal{W}_{x_{1}, \ldots, x_{k}, y_{1}, \ldots, y_{l} ; b}^{a}(\lambda)$ (again after perturbation). These moduli spaces are defined in a way similar to the $\mathcal{M}_{\ldots}(\lambda)$ of $\$ 1$. The starting point consists again of trees as in $\$ 1$ but the root vertex of the tree no longer corresponds to a $J$-disk but rather to a $J$-strip (as in the usual Floer theory) which relates the two intersection points $a, b \in I_{\eta}$. Except for codimension-two phenomena, all of the other vertices correspond to pseudoholomorphic disks with boundaries on one of the $L_{i}$. Moreover, the gradient flows appearing in the construction correspond to one of the two functions $f_{i}$. In short, the elements of these moduli spaces are cluster trees on $L_{0}$ and $L_{1}$ that originate at finite points of the root strip. There may be (finitely) many such clusters attached to the boundary of the strip. These objects are called cluster-strips. It is easy to see how to associate a class $\lambda \in \Pi$ to such an object.

A particular convention is important in dealing with constant strips: among the moduli spaces $\mathcal{W}$ which serve to define our differential, we omit those consisting of the configurations in which the root is the constant strip at some point $a \in I_{\eta}$ and for which there is either no cluster attached to the strip, or more than one.

For generic choices of $J,\left(f_{i}, g_{i}\right)$ and after perturbation, the dimension of the moduli space $\mathcal{W}_{x_{1}, \ldots, x_{k}, y_{1}, \ldots, y_{l} ; b}^{a}(\lambda)$ is

$$
|a|-|b|-\sum\left|x_{i}\right|-\sum\left|y_{j}\right|+\mu(\lambda)-1 .
$$

For one-dimensional moduli spaces, there is a formula analogous to (1.3). As a consequence, we have

Theorem 2.1. With the above notation we have $d_{F}^{2}=0$. The resulting homology is called the fine Floer homology, $\mathbb{F} H_{*}\left(L_{0}, L_{1}, \eta\right)$. Up to isomorphism (and a possible shift in degrees), it does not depend on the choicesmade in its construction, and if 
$\phi: M \rightarrow M$ is a Hamiltonian diffeomorphism, then we have isomorphisms

$$
\mathbb{F} H\left(L_{0}, L_{1}, \eta\right) \simeq \mathbb{F} H\left(\phi\left(L_{0}\right), L_{1}, \eta^{\prime}\right),
$$

where $\eta^{\prime}$ corresponds to $\eta$ via the Hamiltonian diffeomorphism.

Verifying $d_{F}^{2}=0$ is less immediate than for the cluster differential because, besides the usual breaking of clusters and strips, there is a third potential way for boundary points to emerge: they correspond to some cluster tree attached to a strip at some moving point $p$ which "slides" along the boundary of the strip to one of the ends of the strip. There are two reasons that make these boundary components disappear; one is purely algebraic and is a cancellation resulting from our graded commutative setting, and the other is analytic and consists in the fact that (as remarked by Oh [12]) the usual gluing argument applies (under generic conditions) to a $J$-disk passing (transversally) through $a$ and to $a$ itself viewed as a constant strip.

Remark 2.2. For any $\xi \in \tilde{\Gamma}_{\eta}$, we can define its action

$$
\mathcal{A}(\xi)=-\int(u)^{*} \omega
$$

where $u:[0,1] \rightarrow \Gamma_{\eta}$ satisfies $u(0)=\eta, u(1)=\pi(\xi)$ and belongs to the homotopy class defined by $\xi$. As mentioned before, by using an appropriate reparametrization, each element of the moduli spaces $\mathcal{W}_{\ldots ; b}^{a}(\lambda)$ may be viewed as a strip joining $\bar{a}$ and $\bar{b} e^{\lambda}$ (recall that $\bar{a}, \bar{b} \in \tilde{\Gamma}_{\eta}$ are the fixed lifts of the elements $a, b \in I_{\eta}$ ). Clearly, this implies that if $v \in \mathcal{W}_{\ldots ; b}^{a}(\lambda) \neq \emptyset$, then

$$
\mathcal{A}(\bar{a})-\mathcal{A}\left(\bar{b} e^{\lambda}\right)=\int v^{*} \omega
$$

and this integral is non-negative and, obviously, equal to the sum of the symplectic areas of the strip and the disks appearing in $v$. Thus, as in the usual Floer theory, the fine Floer complex admits an action filtration.

\section{Symmetrization}

A particular variant of the construction of the fine Floer homology is useful in applications. To describe it, assume that $L_{0}$ equals $L_{1}$ (we denote both Lagrangian submanifolds by $L$ ), and consider a generic time-dependent Hamiltonian $H_{t \in[0,1]}$, with Hamiltonian flow $\phi_{t \in[0,1]}$, and a generic family of compatible almost complex structures $J_{t \in[0,1]}$. Take as generators of the complex the trajectories $I_{\eta}^{H}$ of $\phi_{t}$ starting at time 0 and ending at time 1 on $L$. We may choose the generic family $J_{t \in[0,1]}$ so that $J_{0}=J_{1}$ (we will denote this almost complex structure by $J$ ).

The symmetric fine Floer complex appears in this setting by additionally choosing the pair $\left(f_{0}, g_{0}\right)$ equal to the pair $\left(f_{1}, g_{1}\right)$ (we denote both pairs by $\left.(f, g)\right)$. Since we have a differential graded algebra multiplication map

$$
\mathcal{C} \ell(L ; J,(f, g)) \otimes \mathcal{C} \ell(L ; J,(f, g)) \rightarrow \mathcal{C} \ell(L ; J,(f, g))
$$

and because $J_{0}=J_{1}=J$, we may replace the ring $\mathcal{R}=\left(\mathcal{C} \ell\left(L_{0} ; J_{0},\left(f_{0}, g_{0}\right)\right) \otimes\right.$ $\left.\mathcal{C} \ell\left(L_{1} ; J_{1},\left(f_{1}, g_{1}\right)\right) \otimes \bar{\Lambda}\right)^{\wedge}$, which appears naturally in the definition of the fine Floer complex, by the ring $\hat{\mathcal{R}}=\left(\mathcal{C} \ell(L ; J,(f, g)) \otimes_{\Lambda} \bar{\Lambda}\right)^{\wedge}$. To define a differential on

$$
\hat{\mathcal{R}} \otimes \mathbb{Q}\left\langle I_{\eta}^{H}\right\rangle,
$$


we use moduli spaces consisting of configurations in which the root pseudoholomorphic strip is replaced by a semicylinder satisfying the usual $(J, H)$-Floer type equation, with its two ends coinciding with trajectories $\gamma, \gamma^{\prime}$ and the two side boundaries lying on $L$.

We denote by $\left(\hat{\mathbb{F}} C(L ; H, J,(f, g)), d_{\hat{F}}\right)$ this symmetric fine Floer homology. If no additional notation appears, the path $\eta$ used in this case is just the constant path.

This homology has the same type of invariance properties as the non-symmetric version, and moreover, it is independent of the choice of $(H, J,(f, g))$ as long as it remains generic.

The advantage of this construction is that it relates directly to the cluster complex as we shall see in the following.

3.1. A little of algebra. Consider a commutative, differential graded algebra of the form $\mathcal{A}=(S V, d)$ with $V$ a rational vector space. Write the elements of the $S V$-module $S V \otimes V$ in the form

$$
x_{1} \cdots x_{k} \otimes v=x_{1} \cdots x_{k} \bar{v}
$$

where $v, x_{i} \in V$. Define the linear map

$$
\alpha: S V \rightarrow S V \otimes V
$$

by letting $\alpha(v)=\bar{v}, \alpha(1)=0$, where 1 is the unit in $S V$, and extending this map by the formula

$$
\alpha(a b)=a \alpha(b)+(-1)^{|a||b|} b \alpha(a) .
$$

Induction on the length of words easily shows that this map is well defined and that the formula above holds for all homogenous monomials $a, b$. Explicitly, we have

$$
\alpha\left(x_{1} \cdots x_{k}\right)=\sum_{i}(-1)^{\sigma_{i}} x_{1} \cdots \hat{x}_{i} \cdots x_{k} \bar{x}_{i},
$$

where $\sigma_{i}$ is the product of the degree of $x_{i}$ and the sum of the degrees of the $x_{j}, j>i$. Define the map $d$ on $S V \otimes V$ as the unique $(S V, d)$-module extension of

$$
d \bar{v}=\alpha(d v)
$$

that satisfies the standard graded Leibniz rule.

One easily checks that $d$ so defined is a differential and that $\alpha$ is a chain map. Denote by

$$
\widetilde{\mathcal{A}}=(S V \otimes V, d)
$$

the $\mathcal{A}$-differential module constructed in this way.

3.2. String-strip symmetrization. We return to our geometric setting. The algebraic construction above appears in the next proposition.

Proposition 3.1. The symmetric fine Floer homology satisfies

$$
s^{-1} \hat{\mathbb{F}} H_{*}(L) \simeq H_{*}(\widetilde{\mathcal{C} \ell}(L ; J,(f, g))) .
$$

This isomorphism is proved in two steps. First, a chain complex $\mathcal{C}$ is constructed by using, instead of root semicylinders, $(J, h)$-linear clusters: they consist of pairs of critical points of the additional Morse function $h$ related via a linear sequence of negative flow lines of $h$ and $J$-disks. Of course, there are still $f$-clusters attached to this linear cluster. This construction is possible even when $f=h$, and in that 
case the resulting complex is precisely (the suspension of) $\widetilde{\mathcal{C} \ell}(L)$. The second step is to define a Piunikhin-Salamon-Schwarz 13] map that relates the complexes $\hat{\mathbb{F}} C_{*}(L, H)$ and $\mathcal{C}$ and prove that it induces an isomorphism in homology.

An immediate corollary of this proposition gives the description of the symmetric fine Floer homology if no bubbling is present.

Corollary 3.2. If $\left.\omega\right|_{\pi_{2}(M, L)}=0$, then

$$
\hat{\mathbb{F}} H_{*}(L) \simeq\left(S\left(s^{-1} H_{*}(L ; \mathbb{Q})\right) \otimes \Lambda\right)^{\wedge} \otimes H_{*}(L ; \mathbb{Q}) .
$$

Clearly, if a Lagrangian is displaceable, then both the fine Floer homology and its symmetric version vanish.

\section{Applications}

We will describe three consequences of our theory.

4.1. The Gromov-Sikorav problem. As a first consequence, we analyze a plausible conjecture going back to Gromov's original paper [8] on pseudoholomorphic curves, stated orally by Sikorav in the late 1980s in the following way: given any compact Lagrangian submanifold of $\mathbb{C}^{n}$, there is a holomorphic disc passing through each point of $L$.

Corollary 4.1. Let $L \subset M$ be a compact, orientable, relative spin Lagrangian submanifold of a symplectic manifold $M$. Assume that $\hat{\mathbb{F}} H_{*}(L)=0$ (for example, if $L$ is displaceable by a Hamiltonian isotopy). Then, for any generic almost complex structure $J$ compatible with the symplectic form, one of the following holds:

i. There are J-holomorphic disks with boundary on L passing through a dense subset of points of $L$.

ii. The cluster complex $\mathcal{C} \ell(L, J, f)$ has high free terms for some Morse function $f$ with a single local minimum and a single local maximum (in particular, there are $J$-disks of non-positive Maslov index).

Proof. Assume that for any Morse function with a single local minimum and local maximum, the associated cluster complex does not have high free terms. Fix such a function $f$ and denote its minimum by $m$. By Proposition 3.1, $s^{-1} \hat{\mathbb{F}} H_{*}(L)$ is isomorphic to $H_{*}(\widetilde{\mathcal{C} \ell}(L, J ;(f, g)))$, which means that $H_{*}(\widetilde{\mathcal{C} \ell}(L, J ;(f, g)))$ vanishes. Note that if $d \bar{m} \neq 0$, then we also have $d m \neq 0$ in the cluster complex. Assume now that

$$
\bar{m} \in \widetilde{\mathcal{C} \ell}(L, J ;(f, g))
$$

is a cycle. Using Remark 1.2, we see that $\bar{m}$ can be a boundary only if $\mathcal{C} \ell(-)$ has free terms: indeed, by this remark, the only possible primitive of $\bar{m}$ must have the form $\tau \bar{m}$, where $\tau$ is a primitive of the unit 1 in the cluster complex. This means that there is a free term in some $d x$ for some $x \in \operatorname{Crit}(f)$. Once again by Remark 1.2. the index of this $x$ cannot be $n$ and it cannot be strictly between 0 and $n$ by our assumption. Therefore, $x=m$ and $d m \neq 0$.

The fact that $d m \neq 0$ means that there exists a non-empty moduli space ${ }^{\nu} \mathcal{M}_{x_{1}, \ldots, x_{k}}^{m}(\lambda)$ of dimension 0 . But for a cluster tree to originate at the minimum $m$, the root disk must go through $m$. As we may use a different function $f$ to place $m$ at any generic point in $L$, this implies the claim. 
The dichotomy in the statement of the previous corollary can be sometimes resolved by homological restrictions.

Corollary 4.2. Suppose that $L$ is orientable and relative spin, and that $H_{2 k}(L ; \mathbb{Q})$ $=0$ for $2 k \notin\{0, \operatorname{dim}(L)\}$. If $\hat{\mathbb{F}} H_{*}(L)=0$, then $L$ satisfies Corollary $4.1 ;$.

Example 4.3. The homological restriction in the previous corollary is serious, but still, there are many examples of such manifolds; $S^{1} \times S^{n-1}$ and its connected sums with itself provide perhaps the simplest examples.

Using symmetrization, we can improve these results in the displaceable case by bounding from above the area of the disks detected in terms of the displacing energy. This upper bound and Gromov's compactness theorem then imply

Corollary 4.4. Suppose that the relative spin, orientable Lagrangian submanifold $L$ is displaceable by a Hamiltonian isotopy, and let $E(L)$ be its Hofer displacement energy. Any $\omega$-tame almost complex structure $J$ has the property that one of the following is true:

i. For any point $x \in L$ there exists a J-holomorphic disk of symplectic area at most $E(L)$ whose boundary rests on $L$ and which passes through $x$.

ii. There exists a J-disk of Maslov index at most

$$
2-\min \left\{2 k \in \mathbb{N} \backslash\{0, \operatorname{dim}(L)\}: H_{2 k}(L ; \mathbb{Q}) \neq 0\right\}
$$

and of symplectic area at most $E(L)$.

A nice geometric consequence of this last corollary is that a displaceable Lagrangian satisfying $H_{2 k}(L ; \mathbb{Q})=0$ for $2 k \notin\{0, \operatorname{dim}(L)\}$ has its relative Gromov radius bounded by $(2 E(L) / \pi)^{1 / 2}$ (see Barraud, Cornea [2] for the definition of this notion).

Remark 4.5. In the monotone case Corollary 4.4 can be deduced in a more direct way. We describe one such proof in 4]. A different proof in this case appears in [1].

4.2. Constraints on Maslov indices. The cluster complex setting provides straightforward proofs of various constraints regarding Maslov indices of Lagrangian submanifolds. For instance, it leads to a quick proof of Fukaya's recent result in [6], which we may state in the following general form: if $S^{1} \times S^{n-1}$ admits a Lagrangian embedding in a symplectic manifold in such a way that it is displaceable by a Hamiltonian isotopy, then $\{2, n\} \cap \operatorname{Im}(\mu) \neq \emptyset$ for $n$ even and $\{2,3-n\} \cap \operatorname{Im}(\mu) \neq \emptyset$ for $n$ odd.

4.3. Detection of periodic orbits. In the presence of an orientable relative spin pair of Lagrangian submanifolds $L, L^{\prime}$, we show that, by replacing in the definition of the clustered moduli spaces one (and only one) of the $J$-disks by a pseudoholomorphic cylinder with one boundary on $L$ and the other boundary on $L^{\prime}$, one can construct a chain map

$$
\text { cyl : } \mathcal{C} \ell(L) \rightarrow \mathcal{C} \ell(L) \otimes \mathcal{C} \ell\left(L^{\prime}\right) \otimes \Lambda_{\Phi_{0}},
$$

where $\Lambda_{\Phi_{0}}$ is an appropriate Novikov ring.

This map induces a morphism in homology whose non-triviality is used to detect the existence of periodic orbits of Hamiltonian diffeomorphisms that separate $L$ from $L^{\prime}$. These results generalize considerably those in [7]. 


\section{REFERENCES}

1. P. Albers, On the extrinsic topology of Lagrangian submanifolds, International Mathematics Research Notices 2005, no. 38, 2341-2371. MR2180810

2. J.-F. Barraud, O. Cornea, Homotopical Dynamics in Symplectic Topology, Morse theoretic methods in nonlinear analysis and in symplectic topology, pp. 109-148, Springer-Verlag, 2006.

3. F. Bourgeois, A Morse-Bott approach to contact homology, PhD thesis, Stanford University 2002.

4. O. Cornea, F. Lalonde, Cluster homology, arXiv:math.SG/0508345 August 2005.

5. K. Fukaya, Y-G. Oh, H. Ohta, K. Ono, Lagrangian intersection Floer theory-anomaly and obstruction, Preprint.

6. K. Fukaya, Application of Floer homology of Lagrangian submanifolds to symplectic topology, To appear in the NATO-ASI 2004 volume, Kluwer, 2005.

7. D. Gatien, F. Lalonde, Holomorphic cylinders with Lagrangian boundary conditions and Hamiltonian dynamics, Duke Math. J. 102 (2000), 485-511. MR.1756107 (2002h:53146)

8. M. Gromov, Pseudoholomorphic curves in symplectic manifolds, Invent. Math. 82 (1985), no. 2, 307-347. MR0809718 (87j:53053)

9. H. Hofer, K. Wysocki, E. Zehnder, Polyfolds and Fredholm theory, Preprint 2005.

10. D. McDuff, D. Salamon, J-Holomorphic Curves and Symplectic Topology, AMS Colloquium Publications, Vol. 52, Providence, RI, 2004. MR2045629 (2004m:53154)

11. Y-G. Oh, Relative Floer and quantum cohomology and the symplectic topology of Lagrangian submanifolds, Contact and symplectic geometry (Cambridge, 1994), pp. 201-267, Publ. Newton Inst., 8, Cambridge Univ. Press, Cambridge, 1996. MR1432465 (98a:58032)

12. Y-G. Oh, Floer Cohomology of Lagrangian Intersections and Pseudoholomorphic Disks I, Comm. P. Appl. Math. 46 (1993), 949-994. MR1223659 (95d:58029a)

13. S. Piunikhin, D. Salamon, M. Schwarz, Symplectic Floer-Donaldson theory and quantum cohomology, Contact and symplectic geometry (Cambridge, 1994), pp. 171-200, Publ. Newton Inst., 8, Cambridge Univ. Press, Cambridge, 1996. MR.1432464 (97m:57053)

14. J. Robbin, D. Salamon, The Maslov index for paths, Topology 32 (1993), 827-844. MR.1241874 (94i:58071)

15. M. Schwarz, A quantum cup-length estimate for symplectic fixed points, Invent. Math. 133 (1998), no. 2, 353-397. MR1632778 (99e:58041)

Université de Montréal, Département de Mathématiques et de Statistique, C.P. 6128

Succ. Centre-ville, Montréal H3C 3J7, Québec, Canada

E-mail address: cornea@dms.umontreal.ca

Université de Montréal, Département de Mathématiques et de Statistique, C.P. 6128

Succ. Centre-ville, Montréal H3C 3J7, Québec, Canada

E-mail address: lalonde@dms.umontreal.ca 